\title{
THE EFFECT OF SEEKING OPERATION ON QOE OF HTTP ADAPTIVE STREAMING SERVICES
}

\author{
Toshiro Nunome and Hiroaki Tani ${ }^{1}$ \\ ${ }^{1}$ Graduate School of Engineering, Nagoya Institute of Technology, Nagoya, Japan
}

\begin{abstract}
In this paper, we assess multidimensional QoE (Quality of Experience) of HTTP-based streaming services in seeking operation to evaluate the effect of two transmission schemes: adaptive bitrate streaming and progressive download. We perform a subjective experiment with two contents and various network load conditions. In the experiment, subjects find pre-specified scenes by means of seeking the video as they want to see the scenes right now. We also perform the principal component analysis for the assessment result of multidimensional QoE. We then find that the adaptive bitrate streaming is not necessarily effective for QoE enhancement; the effectiveness of the scheme depends on the usage of the system and network conditions.
\end{abstract}

\section{KEYWORDS}

Audio and Video Transmission, QoE, Adaptive Bitrate Streaming, Progressive Download

\section{INTRODUCTION}

High quality audiovisual data has been transmitted over the Internet owing to the acceleration of transmission speed of communication networks. Multimedia streaming is one of the major services using audiovisual transmission. Most of the streaming services currently used in the Internet, such as YouTube [1] and Hulu [2], have been provided on a Web browser.

In the past, progressive download has been used as a transmission scheme for the streaming service. However, adaptive bitrate streaming is becoming popular and widely employed [3]. MPEG-DASH (Dynamic Adaptive Streaming over HTTP) [4] is a kind of this scheme. These schemes use HTTP/TCP as a transmission protocol for audio and video [5].

Because the Internet basically provides a best effort service, when a communication line is congested, interruption and latency occur in downloading audio and video data. In such the case, QoS (Quality of Service) degrades, and QoE (Quality of Experience) also degrades. For users, who are recipients of the service, improving QoE is important. Thus, in the streaming service, we need to consider which transmission scheme, the adaptive bitrate streaming or the progressive download, is more effective from a QoE point of view.

Users of the streaming service not only watch video sequentially but also operate the media player freely such as fast forward or rewind of the video. If we can provide the streaming service with high QoE in such operation, the satisfaction of users becomes higher.

There are many studies on QoE of HTTP adaptive streaming services [6], [7]. Most of these studies focus on the effect of bitrate switching on users' subjective quality and do not consider the effect of seeking operation.

DOI: $10.5121 /$ ijcnc.2017.9201 
International Journal of Computer Networks \& Communications (IJCNC) Vol.9, No.2, March 2017

Mok et al. have assessed the relationship between user-viewing activities and QoE [8]. The userviewing activities are pause, resume, refresh, forward time shift, and switch to a higher video quality among others. Then, they have found that video impairment can trigger user-viewing activities, notably pausing and reducing the screen size. However, they have not assessed the relationship between user-viewing activities and QoE in the case of using the adaptive bitrate streaming.

Vilas et al. have modeled the user's behavior of a VoD Website [9]. Yu et al. have performed a traffic analysis of a VoD system and that of an IPTV system in terms of characteristics of the user's behavior and content access patterns [10]. However, the papers have not investigated the influence of the seeking operation on the user's QoE.

This paper is an extended version of [28]. The main contribution is the following. We multidimensionally assess QoE in order to clarify the influence of the seeking operation (fast forward, rewind) on QoE when the user watches video with the two transmission schemes: the adaptive bitrate streaming and the progressive download. Furthermore, we measure end-to-end throughput to consider the difference in the transmission schemes from the viewpoint of bandwidth consumption. These results are helpful in improving the user's QoE of the streaming service.

The remainder of this paper is organized in the following fashion. Section 2 introduces the audio and video transmission schemes in the streaming service. Section 3 introduces related work. Section 4 describes the experimental system. Section 5 outlines the method of QoE assessment. Section 6 presents the experimental results. The paper concludes in Section 7.

\section{AUDIO AND VIDEO TRANSMISSION SCHEMES}

Currently, there are several audio and video transmission schemes in the Internet. The download scheme is one of them and was popular in the early Internet. This scheme downloads video files to the local disk and plays the files after the downloading; that is, the scheme cannot play the files while downloading. The user must wait for a long time until the playback starts.

In contrast, three types of transmission schemes which reduce the waiting time before playback are widely used in the audio and video transmission.

\subsection{Streaming scheme}

The streaming scheme can watch a video while receiving it unlike the download scheme. The scheme receives the video from a streaming server. The streaming server uses a dedicated protocol for transmission. As the dedicated protocols, RTMP (Real-Time Messaging Protocol) [11] and MMSP (Microsoft Media Server Protocol) [12] are employed. MMSP and RTMP can use UDP or TCP as a transport layer protocol. As the dedicated streaming servers, there are Windows Media Server and Flash Media Server.

\subsection{Progressive download}

The progressive download is a combined scheme of the download scheme and the streaming scheme. This scheme can also begin playback while downloading, though this scheme downloads the video file as well as the download scheme.

The Web server uses HTTP/TCP to transmit video data. Unlike the streaming scheme using the dedicated streaming server, this scheme can be widely used by means of the Web server. 
This scheme usually transmits video files of a certain bitrate. For this, though the scheme employs the playout buffer, playback can be paused when a communication bandwidth becomes narrow. In terms of the user-viewing activities, because this scheme plays video from where the download is finished, the user can seek to the position immediately where the video data is already downloaded in the playout buffer.

\subsection{Adaptive bitrate streaming}

The adaptive bitrate streaming separates delivered video into small pieces. They are called as segments, chunks, fragments, and so on. We call them segments in this paper. The length of segments is typically between 2 and 10 seconds. The Web server also uses HTTP/TCP to transmit data.

The segment can be encoded at multiple bitrates, so that the bitrate of the video can be adjusted by network conditions. If the connection quality drops, the client requests the segments encoded at lower bitrates to the server. Conversely, when the communication quality becomes better, the client requests the segments at higher bitrates.

In addition, when the user wants to seeks a scene, the client requests segments related to the scene by sending a HTTP request message. The client can download only required segment and then can seek more freely.

\section{RELATED WORK}

As we mentioned in Section 1, there are many studies on QoE of the HTTP adaptive streaming. However, to the best of our knowledge, no study is available on QoE of the HTTP adaptive streaming in seeking operation.

Reference [6] provides a rigorous survey on QoE issues in the HTTP adaptive streaming based on subjective user tests. It discusses that initial delay, stalling (re-buffering), and bitrate switching are important factors affecting QoE. However, the seeking operation is not considered in the studies discussed in [6]; the authors say that the QoE related to video browsing is currently not investigated in the research yet. In the seeking operation, the effect of the former two factors (i.e., initial delay and stalling) can be changed from normal viewing situations. In the HTTP adaptive streaming, there are three dimensions for adaptation: video frame rate, resolution, and quantization. In this paper, we employ quantization for adaptation because of simplicity.

Reference [7] refers to the switch of representation for adaptation in the HTTP adaptive streaming as quality switch. The authors then survey researches on the effect of quality switch. In addition to the dimensions in [6], they consider the audio bitrate for adaptation. They discuss the effect of the frequency and amplitude of quality switches on subjective QoE. The subjective test designs are very diverse. However, they do not pick up the study treating seeking operation.

In [13], Shen et al. consider not only the average bitrate but also the bitrate fluctuation as the major factors affecting QoE of DASH videos. They propose a QoE model considering the two factors. The quality switch can be assumed as an irregular pattern of bitrate fluctuation. The seeking operation, which they do not deal with, can also cause bitrate fluctuation; it will be different from the fluctuation by quality switch.

Shi et al. perform a viewing engagement pattern modeling in DASH video by means of a machine learning method in [14]. They employ the play percent as an engagement metric. The play percent 
International Journal of Computer Networks \& Communications (IJCNC) Vol.9, No.2, March 2017

is the percent of play time in whole video. The play time is the duration of a session and is calculated by the downloaded chunks. The seeking operation can be considered as user engagement; however, the play percent cannot include the effect of the operation.

\section{EXPERIMENTAL SYSTEM}

Fig. 1 illustrates the experimental system. It consists of four PC's (Web Server, Web Client, Media Server and Media Receiver) and two routers (Cisco 2811 by Cisco Systems). The link between each PC and router is a full duplex Ethernet channel of $100 \mathrm{Mb} / \mathrm{s}$. The link between two routers is a full duplex Ethernet channel of $10 \mathrm{Mb} / \mathrm{s}$.

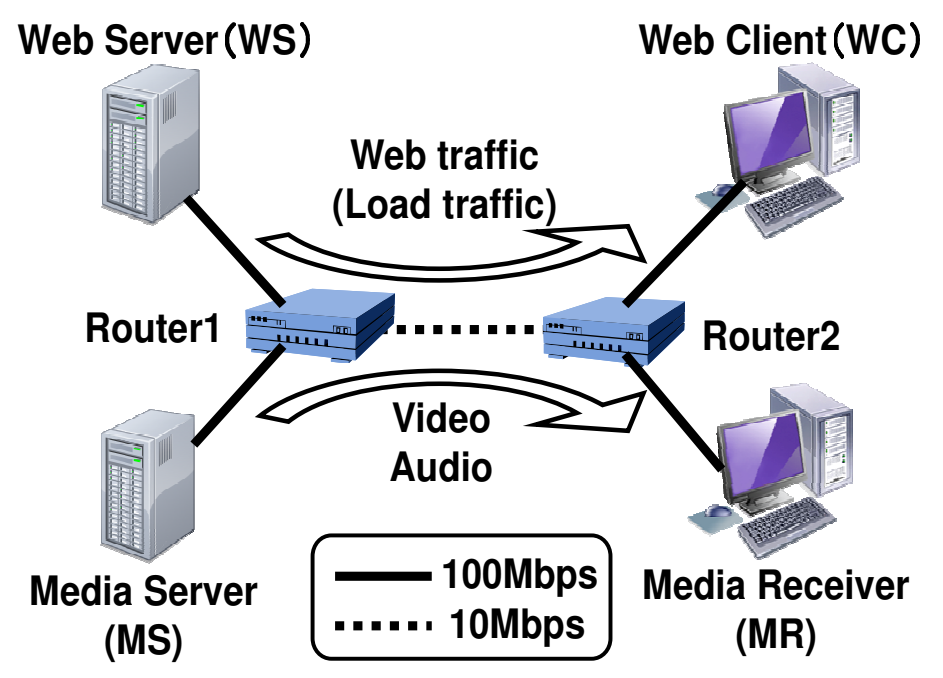

Figure 1. Experimental system

The Media Server (MS) transmits audio and video towards the Media Receiver (MR). The Web Server (WS) transmits the load traffic towards the Web Client (WC). The load traffic is generated by WebStone 2.5 [15]; it is a Web server benchmark tool. WebStone creates a load on a Web server by simulating the activity of multiple clients, which are called Web clients and which can be thought of as users, Web browsers, or other software that retrieves files from a Web server. To create various network conditions, we set the seven patterns of the number of Web clients to Webstone: 0, 10, 20, 25, 35, 40 and 45. The operating system of MS and that of MC are CentOS 6.2. In addition, MS and WS use Apache 2.2 [16] for transmitting data.

We perform the adaptive bitrate streaming by means of HDS (HTTP Dynamic Streaming) [19]. As the media player, we use Strobe Media Playback [20]. In the progressive download, this media player can see how much data have been downloaded in the buffer. The dark blue bar shows where user watches and the light blue bar shows how much video data has stored into the playout buffer. 
Table 1. Audio and video specifications

\begin{tabular}{|l|c||c|}
\hline \multirow{4}{*}{ Audio } & Encoding scheme & AAC-LC \\
\cline { 2 - 3 } & Average bitrate [kbps] & 128 \\
\cline { 2 - 3 } & Channel & stereo \\
\cline { 2 - 3 } & Sampling rate $[\mathrm{kHz}]$ & 48 \\
\hline \multirow{4}{*}{ Video } & Encoding scheme & H.264/AVC \\
\cline { 2 - 3 } & Frame rate [fps] & 29.97 \\
\cline { 2 - 3 } & Picture pattern & I+14P's \\
\cline { 2 - 3 } & Average bitrate [kbps] & $500,1000,1500$ \\
\cline { 2 - 3 } & Resolution & $640 \times 360$ \\
\cline { 2 - 3 } & Container & MP4 \\
\cline { 2 - 3 } & Segment length [s] & 4 \\
\cline { 2 - 3 } & Playing time [s] & 360 \\
\hline
\end{tabular}

Table 1 shows the specifications of audio and video used in the assessment. We employ voaacenc [17] for encoding audio to AAC-LC (Advanced Audio Coding Low Complexity). We use x264 [18] for encoding video to H.264/AVC. In the progressive download, we used two types of videos whose bitrates are $500 \mathrm{kbps}$ and $1500 \mathrm{kbps}$. In the adaptive bitrate streaming, we encoded the video into three types: $500 \mathrm{kbps}, 1000 \mathrm{kbps}$ and $1500 \mathrm{kbps}$. The bitrates in this paper are selected as one of typical cases of the adaptive bitrate streaming. The assessment with more diverse bitrates is one of future study issues. Depending on the load condition of the network, the adaptive bitrate streaming can change the bitrate of segments seamlessly. We set the segment length to 4 seconds, which is the default value.

\section{QOE ASSESSMENT}

\subsection{Task}

In the experiment, we ask subjects to find the pre-specified scenes. The subjects watch the video and seek the scenes. We prepare 42 sheets of A4 papers printed the scenes. Four different scenes are drawn on each paper. They use a sheet of paper in an experimental run. Fig. 2 shows an image of the paper used in the experiment.

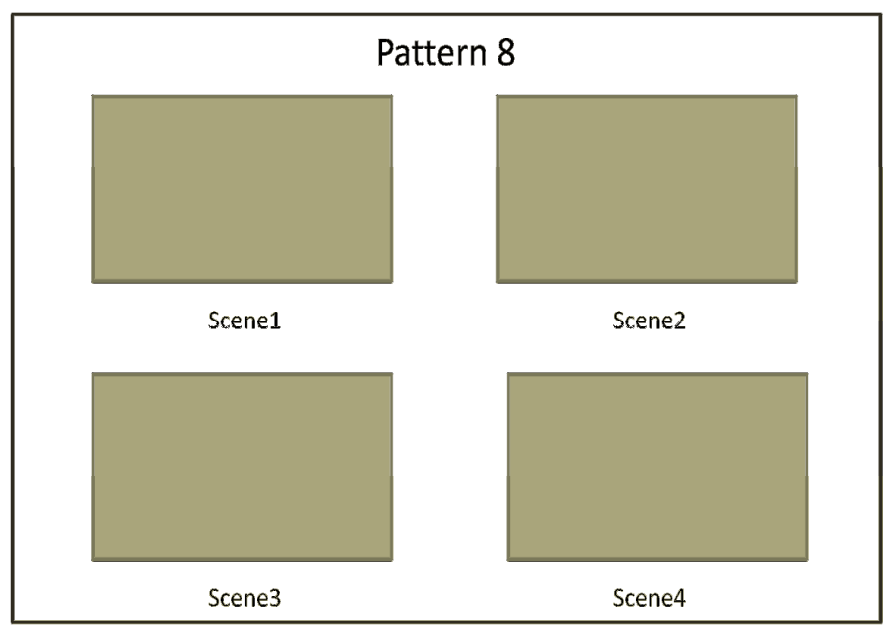

Figure 2. Image of the paper used in the experiment 
There are many kinds of video content. Users' preferences of seeking scenes can be differ for each content. As a sample case for QoE assessment, we use two content types: music video and sport. We use 20 different scenes for each content. We explain the two contents below.

$\cdot$ music video

A concert is held by a male band. The vocal sings songs and the other members play musical instruments. The scenes are entirely dark.

$\cdot$ sport

A football match is taken place. There is a scene such as a free kick, goal, and so on. We can hear the voice of the commentators and cheers of the audience. The scenes are entirely bright.

\subsection{QoE Assessment Method}

We have totally 42 objects to be evaluated because of the two contents, the three transmission schemes, and the seven patterns of load traffic. In order to familiar with the experiment, the subject practices the evaluation with a dummy object at the beginning of each content. We use the progressive download of $1500 \mathrm{kbps}$ in the practice. The half of the subjects begins the experiment with music video. The others begin with sport. The duration of an experimental run is 60 seconds, and the duration for evaluation of the experimental run is 30 seconds. The total time for the experiment to a subject is about 65 minutes.

For multidimensional QoE assessment, we assess an object for evaluation, which is referred to as a stimulus, from many points of view with many pairs of polar terms. A pair of polar terms consists of one adjective and its opposite one, e.g., warm and cool. Table 2 shows the eight polar terms for the experiment. These terms can be classified into four classes: Video, Audio, Task and Psychology. We referred to [21] and [22] to select polar terms. Note that this experiment was performed in Japanese. This paper has translated the used Japanese terms into English. Therefore, the meaning of adjectives written in English here may slightly differ from those of Japanese one.

Table 2. Pairs of polar terms

\begin{tabular}{|c|c|}
\hline class & polar terms \\
\hline \hline \multirow{2}{*}{ Video } & Video is awkward - smooth \\
\cline { 2 - 2 } & Video is dirty - clean \\
\cline { 2 - 2 } & Video is difficult to see - easy \\
\hline Audio & Audio is unnatural - natural \\
\hline Task & Seeking is difficult - easy \\
\hline \multirow{2}{*}{ Psychology } & I feel frustrated - relaxed \\
\cline { 2 - 2 } & I feel impatient - fulfilled \\
\cline { 2 - 2 } & Bad - Excellent \\
\hline
\end{tabular}


International Journal of Computer Networks \& Communications (IJCNC) Vol.9, No.2, March 2017

For each pair of polar terms, the subject gives a score to the stimulus by the rating-scale method [23] with five grades. The best grade (score 5) represents the positive adjective (the right-hand side one in each pair in Table II), while the worst grade (score 1) means the negative adjective. The middle grade (score 3 ) is neutral.

The QoE measure adopted in this paper is the psychological scale, which is an interval scale in the psychometric methods [23]. Note that the QoE measure mainly used in ITU-T/R recommendations and many of technical papers is the MOS (Mean Opinion Score), which is an ordinal scale. Since the interval scale can represent the human subjectivity more accurately than the ordinal scale, we use the psychological scale instead of MOS.

The interval scale can be calculated by the method of successive categories [24]. We apply the law of categorical judgment to the measurement result by the rating-scale method; it can translate the frequency of the scores obtained by the rating scale method into an interval scale. We have to confirm the goodness of fit for the obtained scale. For a test of goodness of fit, we conduct Mosteller's test [25]. Once the goodness of fit has been confirmed, we use the interval scale as the psychological scale.

In addition, the subjects answer the number of scenes which they found in each experimental run. We employed 20 male students in their twenties as the subjects.

\section{EXPERIMENTAL RESULTS}

\subsection{End-to-end throughput}

In this experiment, we installed Wireshark [26] to the Media Receiver in order to measure the bandwidth consumption. We captured all packets which were sent from the Media Server to the Media Receiver by Wireshark. We then counted the TCP payload size of packets received on the Media Receiver.

In Figs. 3 and 4, as the end-to-end throughput, we show the average of the total amount of TCP payload size received in each experimental run, i.e., one minute.

In these figures, for the number of Web clients larger than 0 in music video, the throughput of adaptive bitrate streaming is the smallest, and that of the progressive download of $500 \mathrm{kbps}$ is the second smallest. Since the data transmitting over the network is the smallest, the adaptive bitrate streaming reduces the bandwidth consumption. Owing to the video data is divided into segments, unlike the progressive download, the adaptive bitrate streaming does not always require all the video data and transmits optimal segments suited to the current bandwidth. Meanwhile, the adaptive streaming exchanges HTTP request and response messages for each segment; it can be a factor for reducing throughput. Therefore, the adaptive bitrate streaming is superior to the progressive download in terms of saving network resources. 


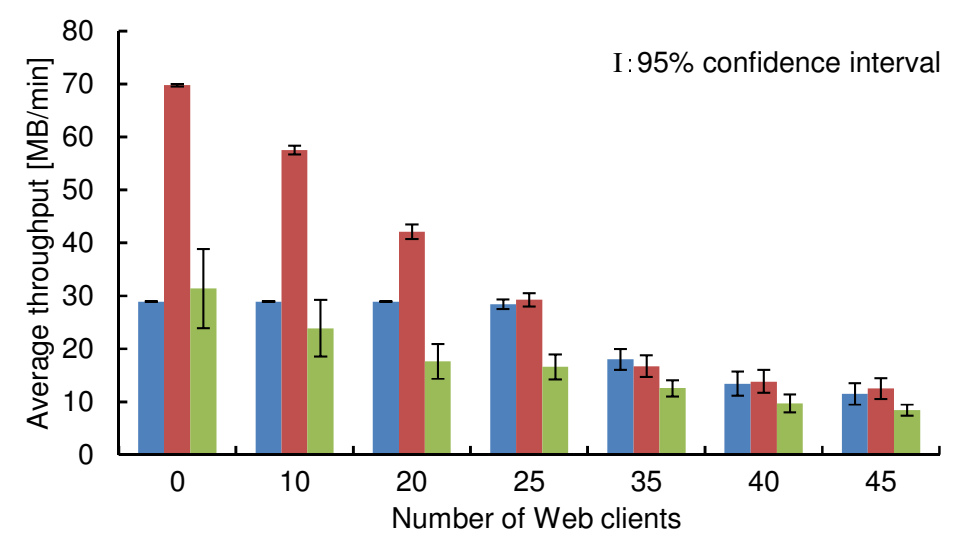

- Progressive Download 500kbps = Progressive Download 1500kbps Adaptive Bitrate Streaming

Figure 3. Average throughput (music video)

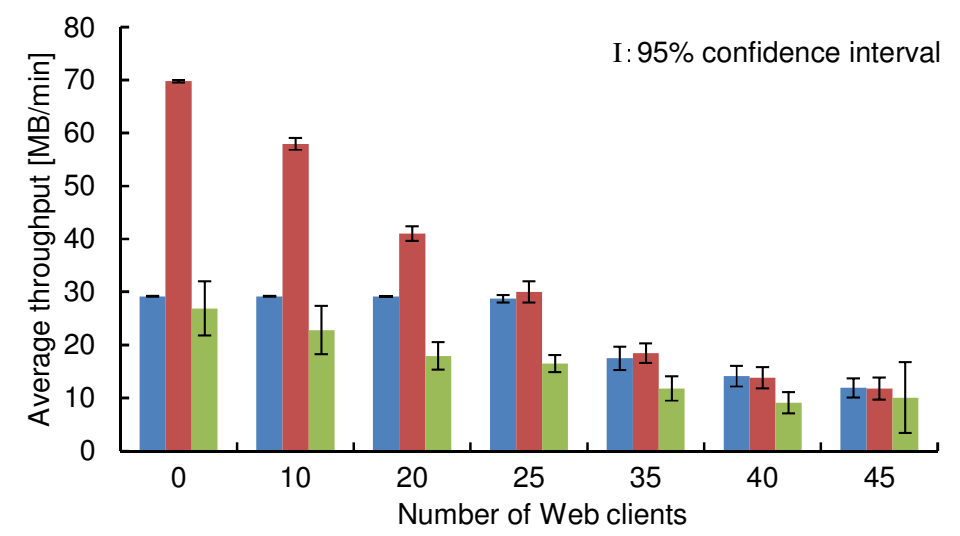

- Progressive Download 500kbps = Progressive Download 1500kbps Adaptive Bitrate Streaming

Figure 4. Average throughput (sport)

The throughput of the progressive download of $500 \mathrm{kbps}$ is roughly constant when the number of Web clients is from 0 to 25 . This is because all the video data are downloaded during 60 seconds. In Fig. 5, we show the average number of segments for each encoding bitrate per minute in the adaptive bitrate streaming in music video. Because there is little difference between the two contents, we present the result for music video only. We notice in the figure that for the number of Web clients smaller than 40, the average number of $1500 \mathrm{kbps}$ segments is larger than the other segments. On the other hand, the number of Web clients is 40 or 45 , the difference between the encoding bitrate becomes small. 


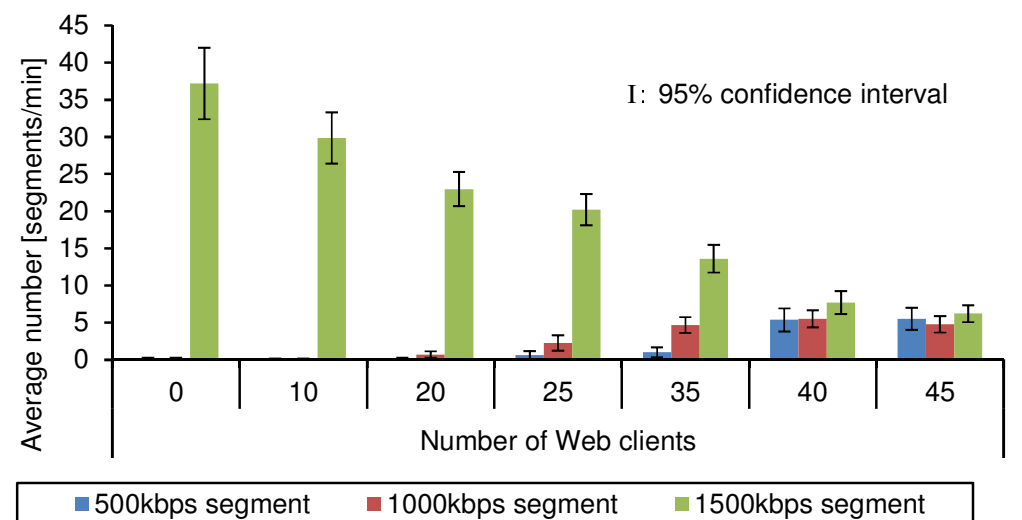

Figure 5. Average number of segments in adaptive bitrate streaming (music video)

\subsection{Psychological Scale}

From the experimental results, we calculated the interval scale for each pair of polar terms. In addition, we carried out Mosteller's test to confirm goodness of fit of the interval scale. As a result, we have found that the hypothesis that the observed value equals the calculated one can be rejected with a significance level of 0.05 in several pairs. Therefore, we removed the stimuli which have large errors until the hypothesis cannot be rejected. In this paper, we use the obtained scale by these processes as the psychological scale. Since we can select an arbitrary origin in an interval scale, we set the minimum value of the psychological scale or the category boundary between categories 1 and 2 to unity for each pair. The maximum value of the psychological scale depends on the distribution of scores by the assessors.

In this paper, we show evaluation results of the polar terms "video is awkward - smooth", "seeking is difficult - easy", "bad - excellent". Figs. 6 and 7 show the psychological scale of "video is awkward - smooth". Figs. 8 and 9 indicate that of "seeking is difficult - easy". Figs. 10 and 11 depict that of "bad - excellent". The dotted lines in the figures indicate the lower limits of Category 2 through Category 5. Note that the results removed by the Mosteller's test are not shown in the figures.

In addition, in Figs. 12 and 13, we show the average number of scenes which the subjects found in an experimental run. There are related to the number of seeking operations while the exact value could not be evaluated because of using the existing media player in the experiment.

\subsection{1. "Video is awkward - smooth"}

When the number of Web clients is 40 and 45 for music video in Fig. 6, and when the number of Web clients is 45 for sport in Fig. 7, the psychological scale values of the adaptive bitrate streaming is higher than that of the progressive download of $1500 \mathrm{kbps}$. On the other hand, the adaptive bitrate streaming has lower psychological scale values than the progressive download of $500 \mathrm{kbps}$, though a few stimuli are removed by the Mosteller's test.

In the progressive download, the subjects cannot move the seek bar of the media player only up to the position where the video data is stored in the playout buffer. In the adaptive bitrate streaming, the subjects can move the seek bar freely without such restrictions; however, it takes time to play the sought scene because re-buffering is caused by reception of a segment containing the data of the sought scene. Thus, the subjects feel awkward because the video pauses. 


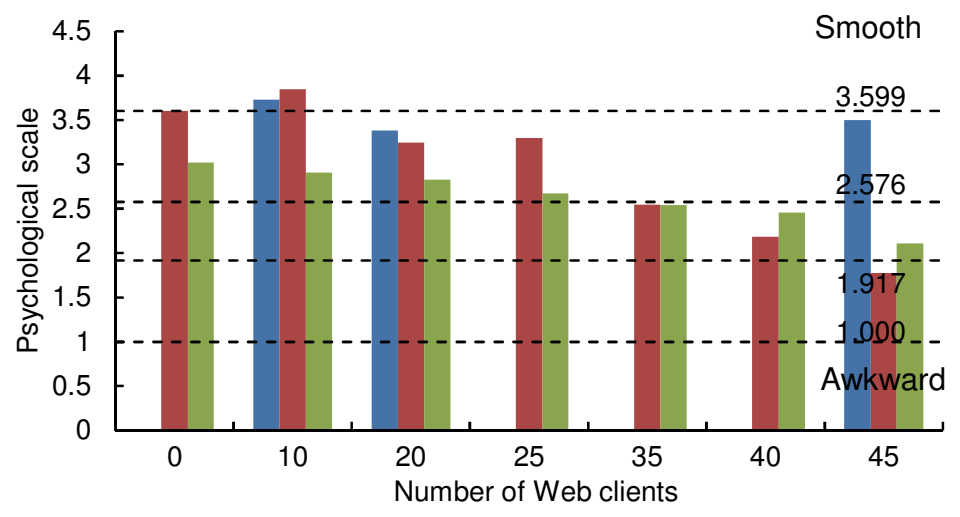

- Progressive Download 500kbps — Progressive Download 1500kbps Adaptive Bitrate Streaming

Figure 6. Video is awkward - smooth (music video)

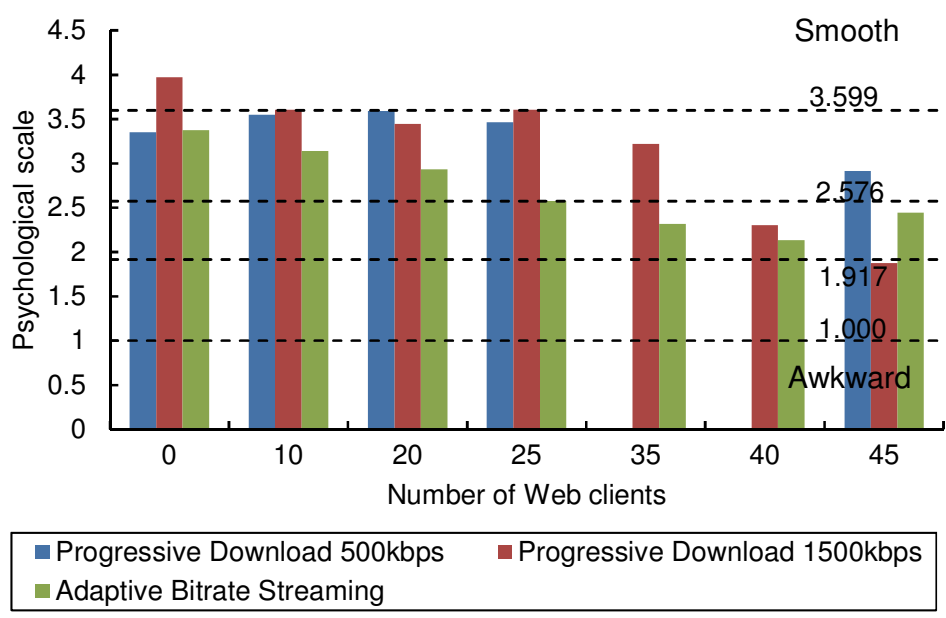

Figure 7. Video is awkward - smooth (sport)

In contrast, in the progressive download, the video is played immediately after seeking the position where the seek bar can be moved. Because the data is likely to be stored in the playout buffer, the effect of pause time is small in progressive download of $500 \mathrm{kbps}$. Therefore, the psychological scale values of the adaptive bitrate streaming are lower than those of the progressive download of $500 \mathrm{kbps}$.

\subsection{2. "Seeking is difficult - easy"}

In Fig. 8, we find that the psychological scale values of the adaptive bitrate streaming are higher than those of the progressive download of $1500 \mathrm{kbps}$ except for the number of Web clients 0 and 10. Furthermore, in Figs. 8 and 9, the psychological scale values of the progressive download of $500 \mathrm{kbps}$ are higher than those of the adaptive bitrate streaming in all the number of Web clients considered here. The reason is as follows. The subjects carry out a lot of seeking operations in this experiment. In Figs. 12 and 13, the progressive download of $500 \mathrm{kbps}$ has the highest number of found scenes in an experimental run. The download time of the progressive download of 500 
kbps is short as described in the discussion for Figs. 3 and 4, and the progressive download of 500 kbps scarcely brings re-buffering. Therefore, the subjects feel easy to seek.

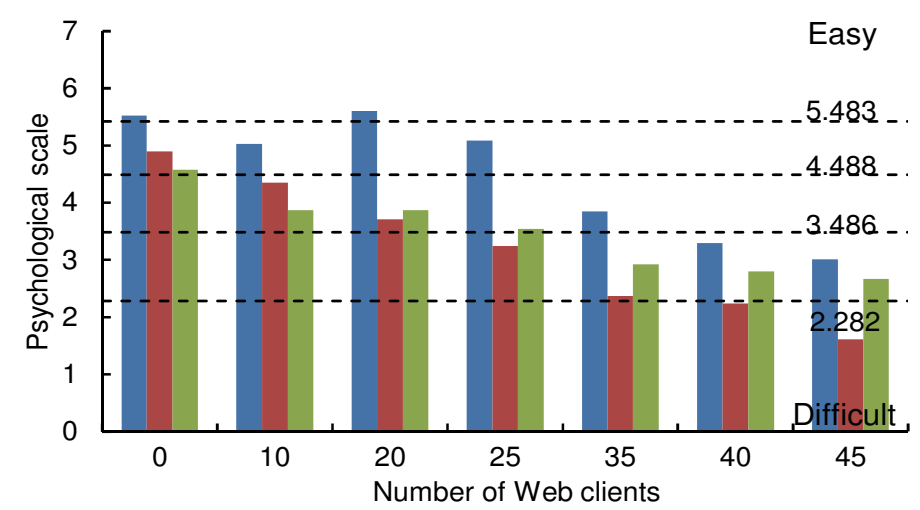

- Progressive Download 500kbps a Progressive Download 1500kbps adaptive Bitrate Streaming

Figure 8. Seeking is difficult - easy (music video)

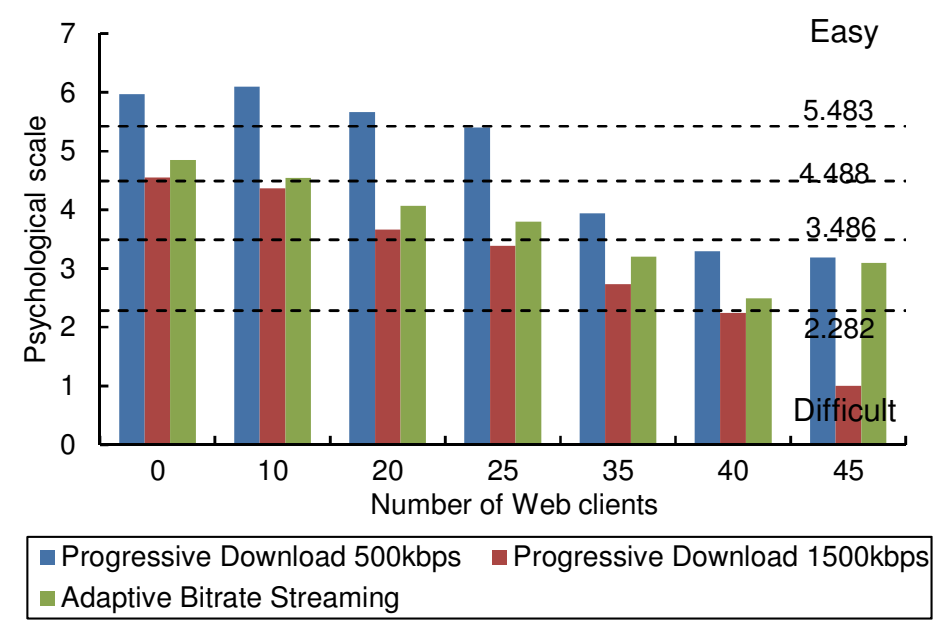

Figure 9. Seeking is difficult - easy (sport)

In the adaptive bitrate streaming, the subjects can move the seek bar freely to a desired scene. However, because re-buffering occurs, the psychological scale values becomes smaller than the progressive download of $500 \mathrm{kbps}$.

\subsection{3. "Bad - Excellent"}

In Figs. 10 and 11, by comparing the adaptive bitrate streaming and the progressive download of $1500 \mathrm{kbps}$, the psychological scale values of the adaptive bitrate streaming is high in the number of Web clients 35 and later. In the progressive download of $1500 \mathrm{kbps}$, the video's bitrate is high, and we can view high-quality video. However, since the data size of the video is large, the range that the subjects can move the seek bar becomes narrow. In the adaptive bitrate streaming, when the available bandwidth becomes narrower, the bitrate of sending video data is adopted because of changing the transmitting segments. In addition, although re-buffering occurs, the subjects can 
seek freely. Therefore, when available bandwidth becomes narrow, the adaptive bitrate streaming is more effective than the progressive download with high bitrate.

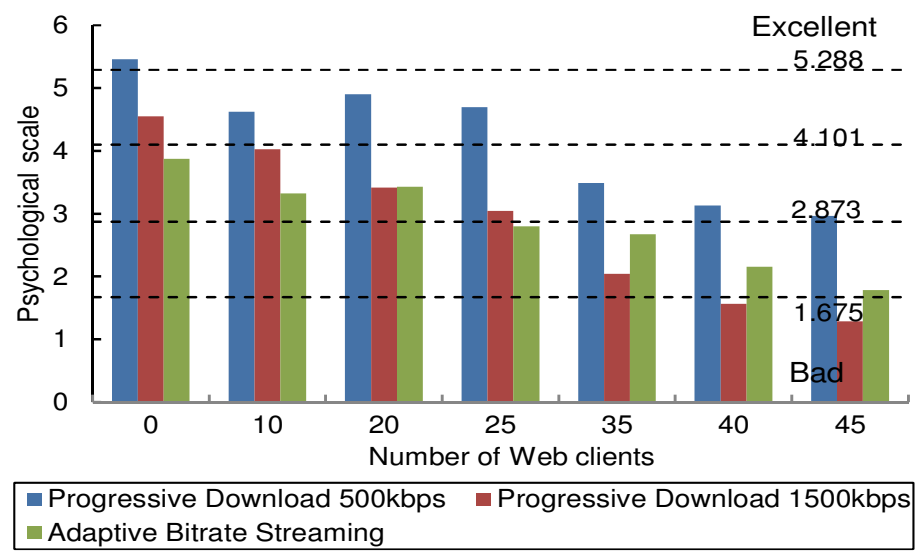

Figure 10. Bad - Excellent (music video)

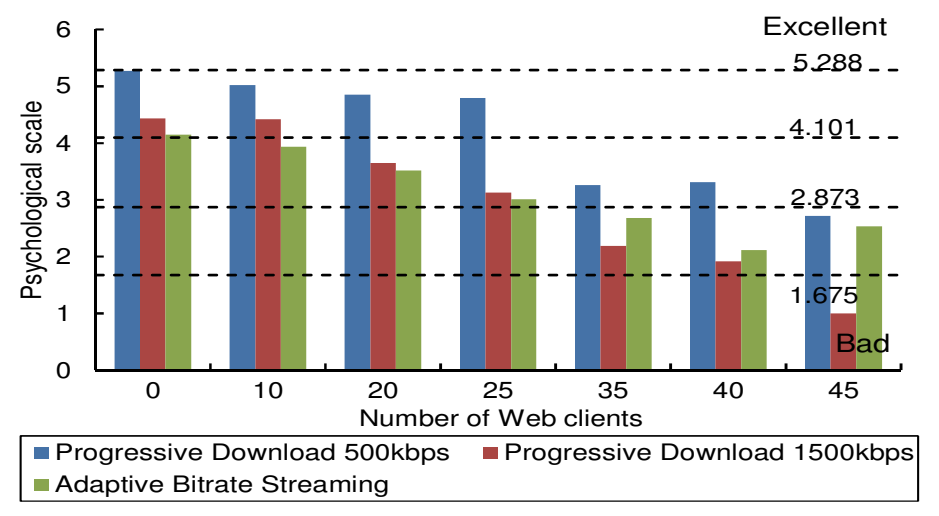

Figure 11. Bad - Excellent (sport)

On the other hand, in these figures, the psychological scale values of the adaptive bitrate streaming are lower than those of the progressive download of $500 \mathrm{kbps}$. Although the adaptive bitrate streaming can transmit high-quality video in accordance with the available bandwidth, we cannot find its advantages in the result.

From the above results, in seeking operations, the adaptive bitrate streaming is not necessarily effective; the effectiveness depends on network conditions. The availability to seek at any time is an advantage of the adaptive bitrate streaming, however, the subjects hate to wait for playback. However, when the available bandwidth becomes narrow, the adaptive bitrate streaming has higher QoE than the progressive download with high bitrate. This is because the adaptive bitrate streaming is easier to seek in such the situation. 


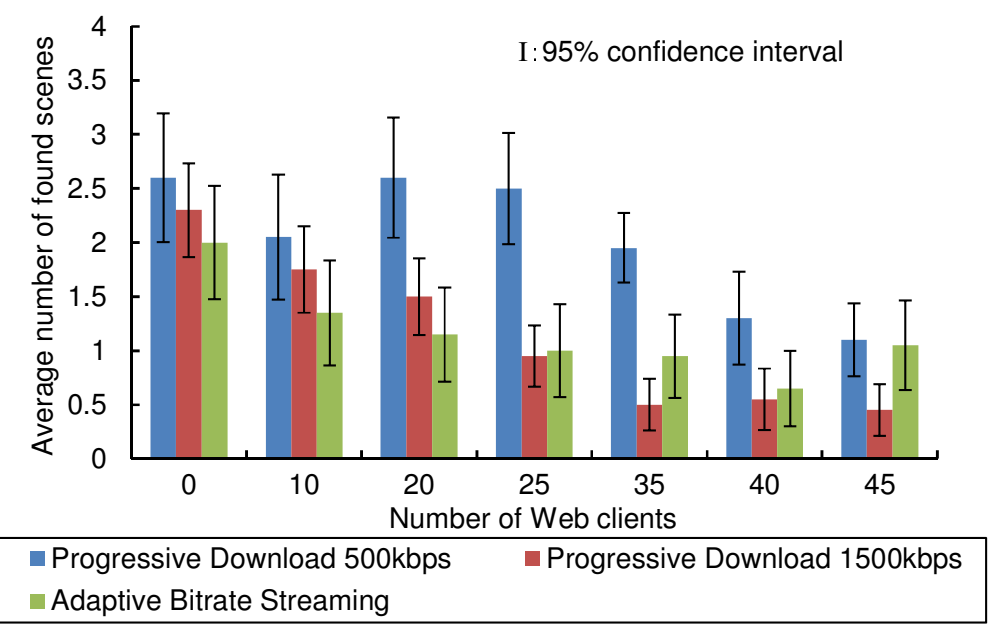

Figure 12. Average number of scenes which subjects found (music video)

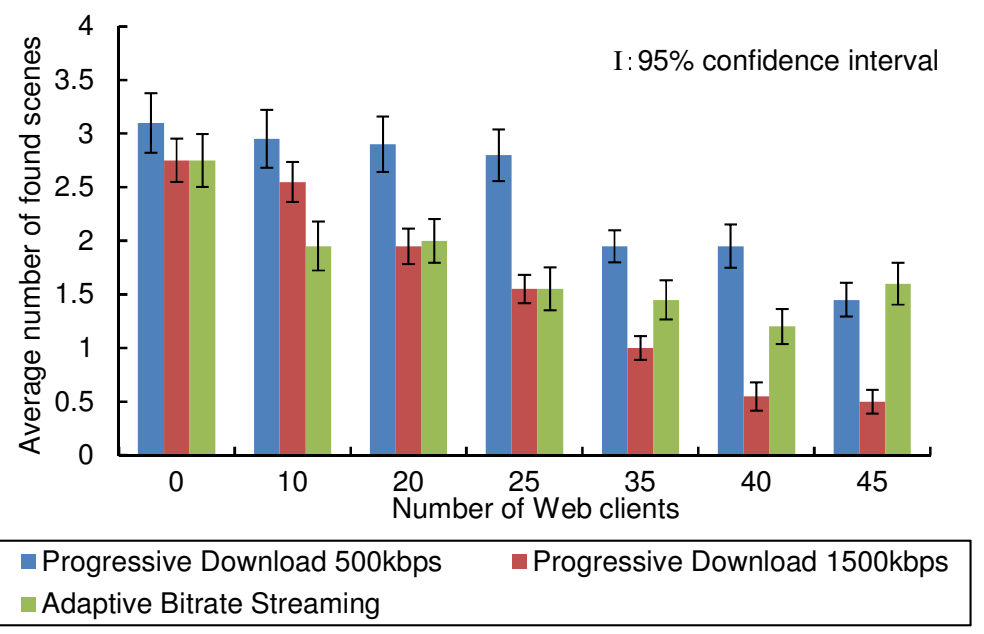

Figure 13. Average number of scenes which subjects found (sport)

\subsection{Principal Component Analysis}

In order to discuss the assessment results deeply, we performed principal component analysis for the five subjective QoE metrics (pairs of polar terms) of Video, Audio, and Task. The principal component analysis is a dimension-reduction technique and to derive a small number of independent linear combinations (principal component) of a set of measured variables [27]. It enables us to understand major factors affecting QoE easily. Each principal component is calculated by taking a linear combination of an eigenvector of the correlation matrix with the variables. The eigenvalues represent the variance of each component.

From the analysis, we picked up two components which have eigenvalues larger than unity. The contribution rate of the first principal component, it is the ratio of the eigenvalue for the first component to the total eigenvalues, is $76.911 \%$. The cumulative contribution rate of the first two principal components is $92.407 \%$. 
International Journal of Computer Networks \& Communications (IJCNC) Vol.9, No.2, March 2017

Fig. 14 plots the principal component loading values. The loading value represents the correlation coefficient between the factor and the QoE measure. The abscissa indicates the first principal component loading, and the ordinate represents the second one. Here, a positive value means a positive relationship between the component loading and the QoE measure, and a negative value presents a negative relationship. We notice in the figure that "video is awkward - smooth" and "video is difficult to see - easy" have large loading values of the first principal component. These pairs represent video temporal quality. In addition, "audio is unnatural - natural" and "seeking is difficult - easy" are close to the above two adjective pairs for video. Thus, when the video temporal quality is good, the users feel that seeking is easy.

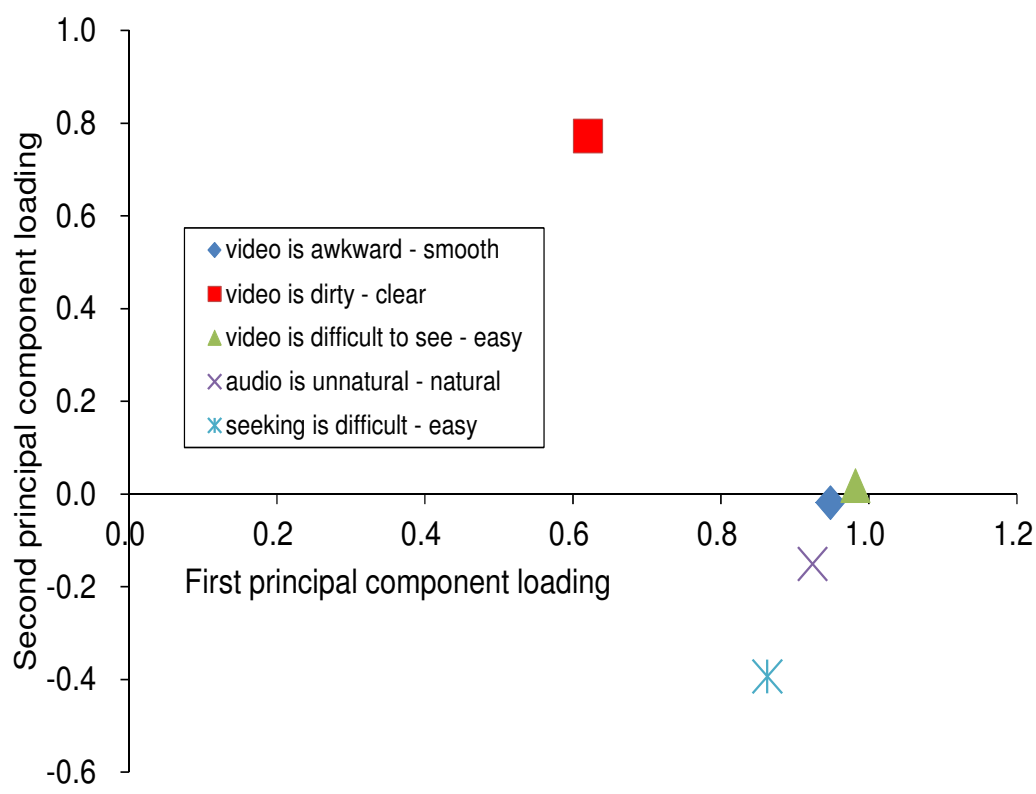

Figure 14. First and second principal component loading values.

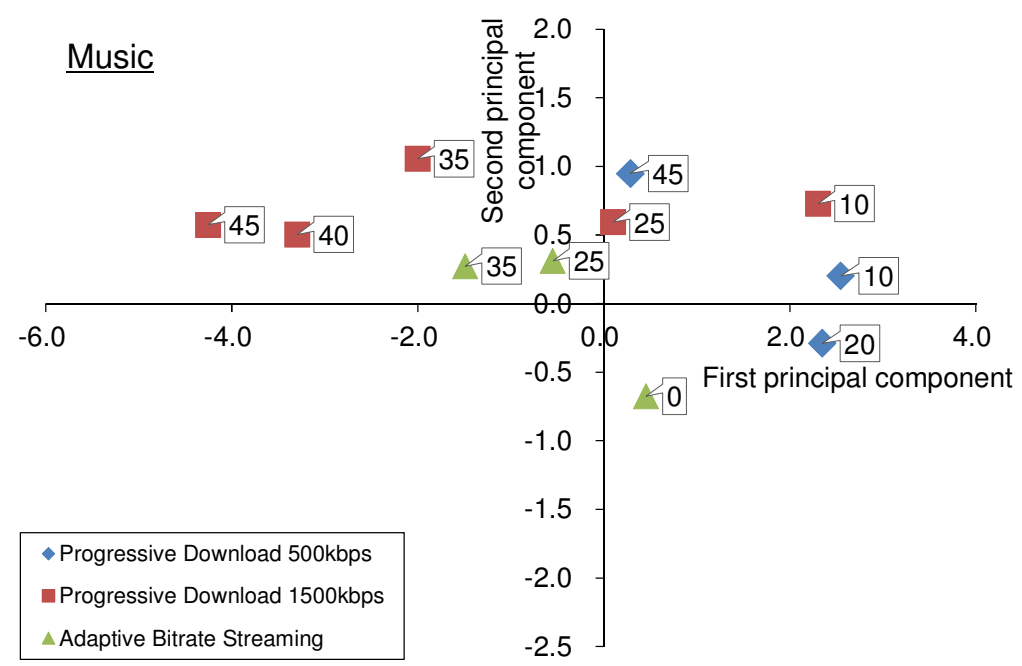

Figure 15. First and second principal component scores (music video). 
International Journal of Computer Networks \& Communications (IJCNC) Vol.9, No.2, March 2017

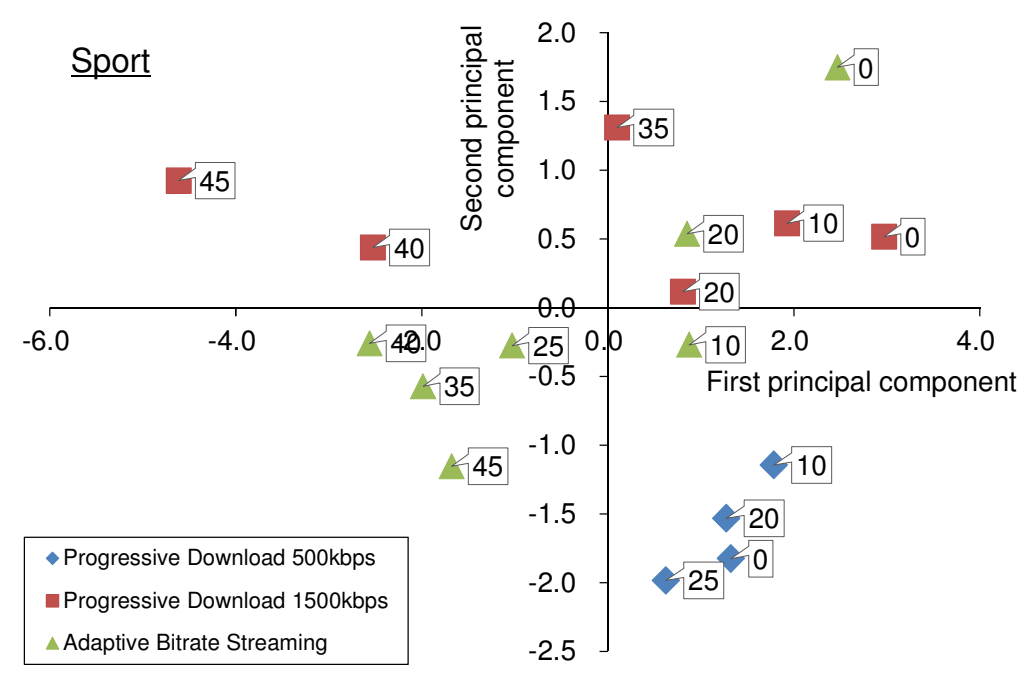

Figure 16. First and second principal component scores (sport).

We also find in Fig. 14 that "video is dirty - clear" has large second principal component loading. It implies that the users recognized the video temporal quality and the video spatial quality separately.

Figs. 15 and 16 show the principal component scores for music video and sport, respectively. The principal component scores are calculated by the factor loadings and standardized psychological scale values for each stimulus. In these figures, the abscissa and ordinate are the first principal component score and the second one, respectively. The balloons accompanying each symbol represent the number of Web clients. The discarded stimuli by means of the Mosteller's test are not shown in the figures.

We find in Figs. 15 and 16 that the first principal component score decreases as the number of Web clients increases. This is because the output quality of video degrades as the background traffic becomes heavy.

In Fig. 16, we see that the second principal component scores for the progressive download 500 kbps are smaller than those for the other methods. This is owing to the low video spatial quality. Sport is a video dominant content. Thus, the effect of the bitrate for the second principal component is larger than that in music video (Fig. 15).

We notice in Fig. 16 that the number of Web clients affects both the first and the second principal component score in the adaptive bitrate streaming, while it affects the first principal component score in the progressive download methods. This represents that the adaptive bitrate streaming can change the video encoding bitrate according to the network condition.

\subsection{Correlation coefficient between the polar terms}

In order to consider the major factors affecting users' overall satisfaction, we calculated the correlation coefficient between "bad - excellent" and one of the other seven polar terms used in the experiment. It measures the strength and direction of a linear relationship between two variables and has a value between +1 and -1 . We show the correlation coefficients higher than 0.9 in descending order in Table 3. 
Table 3. Correlation coefficient with "bad - excellent"

\begin{tabular}{|c|c|}
\hline polar term & correlation coefficient \\
\hline \hline I feel frustrated - relaxed & 0.990 \\
\hline Seeking is difficult - easy & 0.982 \\
\hline I feel impatient - fulfilled & 0.979 \\
\hline
\end{tabular}

In Table 3, we notice that "bad - excellent" has high correlation coefficients with "I feel frustrated - relaxed", "seeking is difficult - easy", and "I feel impatient - fulfilled". In this experiment, the seeking operation has an important role. When the seeking operation becomes difficult, the subjects become frustrated, and the frustration degrades overall quality.

\section{CONCLUSIONS}

We assessed the effect of the transmission schemes in seeking operation on end-to-end throughput and QoE in the Web-based audio and video transmission.

As a result, we confirmed that the adaptive bitrate streaming reduces the bandwidth consumption. Further, we found that the ease of seeking affects the psychology and the overall QoE. When the user of streaming service watches a video while seeking, the adaptive bitrate streaming is not necessarily effective.

As future work, we should devise a method that the effect of re-buffering is small in the adaptive bitrate streaming. This is because re-buffering has a significant impact on QoE. In addition, we should consider the effect of the implementation of the media player. In the progressive download, we used the media player which could not move the seek bar to the position where video data is not stored in the playout buffer. Other implementation can be considered for seeking operation. Furthermore, the player cannot assess the re-buffering time and exact number of seeking operations quantitatively; it is needed to devise a way to evaluate these parameters.

In our subjective experiment, we employed a HTTP adaptive streaming traffic and background HTTP file transfer streams. When multiple HTTP adaptive streaming traffic exists, the traffic suffers stability, fairness, and network utilization problems [6]. We then need to consider the situation in future study.

\section{REFERENCES}

[1] "YouTube," http://www.youtube.com/.

[2] "Hulu," http://www.hulu.com/.

[3] O. Oyman and S. Singh, "Quality of experience for HTTP adaptive streaming services," IEEE Communication Magazine, vol. 50, no. 4, pp. 20-27, Apr. 2012.

[4] I. Sodagar, "The MPEG-DASH standard for multimedia streaming over the Internet," IEEE Multimedia, vol. 18, no. 4, pp. 62-67, April 2011.

[5] A. C. Begen, T. Akgul and M. Baugher, "Watching video over the Web, Part I: streaming protocols," IEEE Internet Computing, vol. 15, no. 2, Mar./Apr. 2011.

[6] M. Seufert, S. Egger, M. Slanina, T. Zinner, T. Hobfeld and P. Tran- Gia, "A survey on quality of experience of HTTP adaptive streaming,” IEEE Commun. Surveys \& Tutorials, vol. 17, no. 1, pp. 469-492, 1Q, 2015.

[7] M. -N. Garcia, F. De Simone, S. Tavakoli, N. Staelens, S. Egger, K. Brunnstrom and A. Raake, "Quality of experience and HTTP adaptive streaming: A review of subjective studies," Proc. QoMEX 2014, pp. 141-146, Sept. 2014.

[8] R. K. P. Mok, E. W. W. Chan, X. Luo and R. K. C. Chang, "Inferring the QoE of HTTP video streaming from user-viewing activities," Proc. ACM W-MUST 2011, Aug. 2011.

[9] M. Vilas, X. Paneda, R. Garcia, D. Melendi and V. Garcia, "User behavior analysis of a video-on-demand service with a wide variety of subjects and lengths," Proc. EUROMICRO, 2005. 
International Journal of Computer Networks \& Communications (IJCNC) Vol.9, No.2, March 2017

[10] H. Yu, D. Zheng, B. Y. Zhao and W. Zheng, "Understanding user behavior in large-scale video-on-demand systems," SIGOPS Oper. Syst. Rev., 40, pp. 333-344, April 2006.

[11] Adobe Systems, "Real-time messaging protocol (RTMP) specification," http://www.adobe.com/jp/devnet/rtmp.html.

[12] Microsoft, "[MS-MMSP]: Microsoft media server (MMS) protocol," http://msdn.microsoft.com/enus/library/cc234711.aspx.

[13] Y. Shen, Y. Liu, H. Yang and D. Yang, "Quality of experience study on dynamic adaptive streaming based on HTTP,” IEICE Trans. Commun., vol. E98-B, no. 1, pp. 62-70, Jan. 2015.

[14] W. Shi, Z. Wang, C. Zhou and L. Sun, "Characterizing viewing engagement patterns in DASH," Proc. IEEE ICME 2015, June/July 2015.

[15] Mindcraft, "WebStone benchmark information," http://www.mindcraft.com/webstone/.

[16] "Apache HTTP server project," http://httpd.apache.org/.

[17] “opencore-amr," http://sourceforge.net/projects/opencore-amr/files/.

[18] “x264,” http://www.videolan.org/developers/x264.html.

[19] Adobe Systems, "HTTP dynamic streaming," http://www.adobe.com/jp/products/hds-dynamic-streaming.html.

[20] "Strobe media playback," http://www.osmf.org/strobe mediaplayback.html.

[21] T. Ichikawa, T. Nunome and S. Tasaka, "Multidimensional assessment and principal component analysis of QoE in interactive multi-view video and audio IP communications," Proc. ICOIN 2012, pp. 482-487, Feb. 2012.

[22] Y. Ito and S. Tasaka, "End-to-end monitoring of multidimensional user- level QoS in audio-video IP transmission," Proc. Fifth IEEE/IFIP Work- shop on End-to-End Monitoring Techniques and Services (E2EMON 2007), pp. 1-8, May 2007.

[23] J. P. Guilford: Psychometric methods, McGraw-Hill, N. Y., 1954.

[24] S. Tasaka and Y. Ito: "Psychometric analysis of the mutually compensatory property of multimedia QoS," Proc. IEEE ICC 2003, pp. 1880-1886, May 2003.

[25] F. Mosteller: "Remarks on the method of paired comparisons: III. a test of significance for paired comparisons when equal standard deviations and equal correlations are assumed," Psychometrika, vol. 16, no. 2, pp. 207-218, June 1951.

[26] "Wireshark," http://www.wireshark.org/.

[27] “Principal Components,” http://www.jmp.com/support/help/Principal_Components.shtml.

[28] H. Tani and T. Nunome, "QoE assessment of a Web-based streaming service in seeking operation," Proc. ICOIN 2014, pp. 254-259, Feb. 2014.

\section{Authors}

Toshiro Nunome received the B.S., M.S. and Ph.D. degrees from Nagoya Institute of Technology, Nagoya, Japan, in 1998, 2000, and 2006, respectively. From 2000 to 2001, he was with Pioneer Corp. In 2002, he joined Nagoya Institute of Technology as a Research Associate. Now, he is an Associate Professor in the Department of Computer Science, Graduate School of Engineering. His research interests include multimedia QoE/QoS, multicast communications and wireless networks. Dr. Nunome is a member of the IEEE, the IEICE and the Institute of Image Information and Television Engineers.

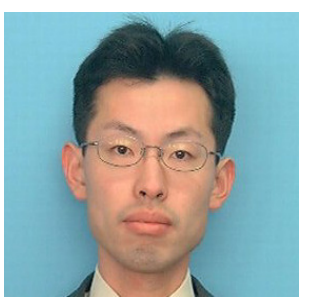

Hiroaki Tani received the B.S. and M.S. degrees from Nagoya Institute of Technology, Nagoya, Japan, in 2013 and 2015, respectively. Currently, he works at NTT DATA i Corp.

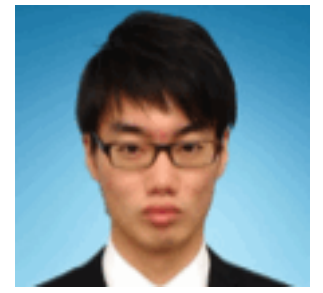

